\title{
Prática mental e equilíbrio em indivíduos após Acidente Vascular Cerebral isquêmico
}

\author{
Mental practice and balance in individuals after \\ ischaemic stroke
}

\section{Práctica mental y equilibrio en personas después de accidente vascular cerebral isquémico}

Janieli Maciel Santana ${ }^{1}$, Mariana Duarte de Sousa ${ }^{2}$, Silviane Henriques Dantas $^{2}$, Eloise de Oliveira Lima ${ }^{3}$

1.Graduanda do curso de Fisioterapia pela UNINASSAU-CG, Campina Grande-PB, Brasil.

2.Fisioterapeutas pela UNINASSAU-CG, Campina Grande-PB, Brasil.

3.Fisioterapeuta, Mestre, Professora do curso de Fisioterapia da UNINASSAU-CG. Campina Grande-PB, Brasil.

\begin{abstract}
Resumo
Introdução. A prática mental (PM) tem sido sugerida como uma possibilidade de terapia auxiliar na reabilitação motora de pacientes com desordens neurológicas. A técnica requer que o paciente mantenha a capacidade de movimento, mas que tenha somente a intenção de realizar alguma tarefa motora. Objetivo. Avaliar a influência da prática mental sobre o equilíbrio de indivíduos com Acidente Vascular Cerebral isquêmico (AVC). Método. Participaram da pesquisa seis pacientes atendidos no setor de neurologia da Clínica Escola da

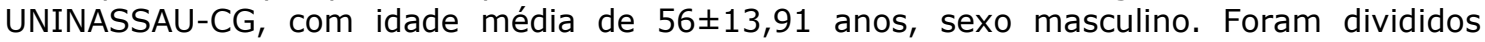
aleatoriamente em dois grupos: grupo que realizou apenas a fisioterapia motora - GFM $(n=3)$ e grupo que realizou a fisioterapia motora e a prática mental - GFM + PM $(n=3)$. Os participantes foram avaliados utilizando o Mini Exame do Estado Mental, Teste de equilíbrio de Berg e Timed Up \& Go. Resultados. Não houve diferença entre GFM+PM e GFM em relação ao equilíbrio $(p<0.05)$. Entretanto, observou-se que os dois grupos apresentaram melhoras, sugerindo que o indivíduo pós AVC pode ser beneficiado na recuperação do equilíbrio e na redução do risco de quedas realizando apenas a fisioterapia motora ou realizando a prática mental associada a fisioterapia motora. Conclusões. Reforça-se a importância da adesão do fisioterapeuta a novas técnicas, de modo a interligar os sistemas envolvidos na ativação de áreas corticais relacionadas a realização do movimento que são de extrema importância para a estimulação da plasticidade neuronal.
\end{abstract}

Unitermos. Cognição; Equilíbrio postural; Acidente Vascular Cerebral

\footnotetext{
Abstract

Introduction. Mental practice (MP) has been suggested as a possibility of auxiliary therapy in motor rehabilitation of patients with neurological disorders. The technique requires that the patient maintain the ability to move, but that he has only the intention of performing some motor task. Objective. To evaluate the influence of mental practice on the balance of individuals with ischemic stroke. Method. Six patients attended at the neurology department of Clinic School of UNINASSAU-CG participated in the research, with a mean age of $56 \pm 13.91$ years, male. They were randomly divided into two groups: group that performed only motor physical therapy - GMP $(n=3)$ and group that performed motor physical therapy and mental practice - GMP + MP $(n=3)$. Participants were assessed using the Mini Mental State Examination, Berg balance test, and Timed Up \& Go. Results. There was no difference between the GMP + MP and GMP in relation to balance $(p<0.05)$. However, it was observed that both groups showed improvements, suggesting that the post-stroke individual can benefit from recovering balance and reducing the risk of falls by performing only motor physical therapy or performing mental practice associated with motor physical therapy. Conclusions.
} 
The importance of the physical therapist adherence to new techniques is reinforced, in order to interconnect the systems involved in the activation of cortical areas related to the movement, which are extremely important for the stimulation of neuronal plasticity.

Keywords. Cognition; Postural balance; Stroke

\section{Resumen}

Introducción. Se ha sugerido la práctica mental (PM) como una posibilidad de terapia auxiliar en la rehabilitación motora de pacientes con trastornos neurológicos. La técnica requiere que el paciente mantenga la capacidad de moverse, pero que solo tiene la intención de realizar alguna tarea motora. Objetivo. Evaluar la influencia de la práctica mental en el equilibrio de las personas con accidente cerebrovascular isquémico. Método. Seis pacientes atendidos en el departamento de neurología de la Clínica Escola da UNINASSAU-CG participaron en la investigación, con una edad media de 56士13.91 años, hombres. Se dividieron al azar en dos grupos: grupo que realizó solo fisioterapia motora - GFM $(n=3)$ y grupo que realizó fisioterapia motora y práctica mental - GFM + PM $(n=3)$. Los participantes fueron evaluados utilizando el Mini Examen del Estado Mental, la prueba de equilibrio de Berg y Timed Up \& Go. Resultados. No hubo diferencias entre los GFM + PM y GFM en relación con el equilibrio $(p<0.05)$. Sin embargo, se observó que ambos grupos mostraron mejoras, lo que sugiere que el individuo después del accidente cerebrovascular puede beneficiarse de la recuperación del equilibrio y la reducción del riesgo de caídas al realizar solo fisioterapia motora o realizar una práctica mental asociada con la fisioterapia motora. Conclusiones. Se refuerza la importancia de la adherencia del fisioterapeuta a las nuevas técnicas, para interconectar los sistemas involucrados en la activación de las áreas corticales relacionadas con el movimiento, que son extremadamente importantes para la estimulación de la plasticidad neuronal.

Palabras clave. Cognición; Equilibrio postural; Accidente cerebrovascular

Trabalho realizado no Centro Universitário Maurício de Nassau, Campina Grande-PB, Brasil.

Conflito de interesse: não

Recebido em: 05/08/2020

Aceito em: $04 / 11 / 2020$

Endereço para correspondência: Eloise O Lima. R. Antônio Carvalho de Souza 295. Estação Velha. Campina Grande-PB, Brasil. CEP 58410-030. E-mail: eloise olima@hotmail.com

\section{INTRODUÇÃO}

O acidente vascular cerebral (AVC) caracteriza-se pela deficiência de sangue no encéfalo devido a uma obstrução ou rompimento de estruturas responsáveis pela irrigação sanguínea. Sendo, dessa forma, classificado em isquêmico ou hemorrágico, respectivamente ${ }^{1}$. A Organização Mundial de Saúde (OMS) estima que até 2030, o AVC continue sendo a segunda maior causa de mortes no mundo. Dados da Sociedade Brasileira de Doenças Cerebrovasculares apontam o Brasil em $1^{\circ}$ lugar nesse índice. O evento pode ocasionar múltiplas sequelas, diante disso, é de fundamental 
importância que a reabilitação seja desenvolvida por uma equipe ${ }^{2}$.

O AVC traz diversas repercussões clínicas pelo fato de ser uma doença incapacitante. Dentre suas consequências são encontradas alterações nas funções sensitiva, motora, cognitiva, perceptiva, comunicativa e emocional que afetam a funcionalidade nas atividades de vida diária, interação social e lazer ${ }^{3}$.

O evento provoca diversas deficiências no Sistema Nervoso Central (SNC), influenciando diretamente no comprometimento do sistema sensorial e redes motoras afetando consequentemente o nível funcional, o equilíbrio, o controle postural e a marcha4.

No indivíduo hemiparético ocorrem alterações no equilíbrio dinâmico e estático que se relacionam com o déficit proprioceptivo, controle de tronco e força. Com a perda parcial da força muscular ocorre um deslocamento do centro de gravidade, transferindo o peso corporal para o lado não parético e aumentando consideravelmente 0 risco de quedas ${ }^{5}$.

Recentemente à prática mental (PM) tem sido sugerida como uma possibilidade de terapia auxiliar na reabilitação motora de pacientes com desordens neurológicas. Trata-se de um método de treinamento que objetiva promover a ativação de áreas cerebrais relacionadas ao movimento, permitindo que movimentos antes acessados de forma inconsciente sejam acessados conscientemente ${ }^{6}$. 
A PM acontece por meio de uma simulação mental com várias repetições, sem que haja a presença de qualquer movimento, sendo elaborada por imagens internas ou externas. A imagem interna tem caráter cinestésico já a imagem externa é puramente visual e pode ser executada tanto na primeira pessoa quanto na terceira. Quando a PM é realizada na primeira pessoa 0 indivíduo imagina 0 movimento sendo realizado por um segmento do seu próprio corpo. Quando feita na terceira pessoa o paciente imagina o movimento sendo realizado por outrem ${ }^{7}$.

Diferente de outras técnicas de reabilitação passivas ou ativas, a PM não requer que o paciente mantenha a capacidade de movimento, mas que tenha somente a intenção de realizar alguma tarefa motora, além disso, é um método de baixo custo que pode aumentar a recuperação acima do nível já alcançado com tratamentos convencionais ${ }^{8,9}$.

Portanto, o objetivo do presente estudo foi avaliar a influência da prática mental sobre o equilíbrio e a marcha de indivíduos com Acidente Vascular Cerebral isquêmico (AVCI).

\section{MÉTODO}

\section{Tipo de estudo}

Tratou-se de um ensaio clínico randomizado do tipo longitudinal, apresentando uma análise descritiva por meio uma abordagem quantitativa, realizado nos meses de agosto a outubro de 2019, na Clínica Escola do Centro Universitário 
Maurício de Nassau, localizada na cidade de Campina Grande-PB, após autorização institucional.

\section{Amostra}

A população foi constituída por 20 indivíduos com AVC e após analisados os critérios de inclusão e exclusão permaneceram na amostra um total de 6 participantes. A amostra foi do tipo não probabilística seguindo o critério de acessibilidade. Foram selecionados pacientes com diagnóstico clínico de AVCI, clinicamente estáveis (acima de 6 meses após o evento), sem déficit cognitivo (comprovado pelo Mini Exame do Estado Mental com escore maior ou igual a 20), ambos os sexos e idade acima de 40 anos e que aceitaram participar da pesquisa como voluntários. Foram excluídos 14 indivíduos por não deambularem.

Após a aprovação do projeto pelo Comitê de Ética em pesquisa da UNIFACISA/CESED (CAAE: 04909718.5.0000.5175) o estudo foi desenvolvido levandose em consideração os aspectos éticos de pesquisa envolvendo seres humanos, preconizados pela Resolução 466/12 do Conselho Nacional de Saúde.

\section{Procedimento}

Realizou- se o convite para a participação da pesquisa aos pacientes que eram atendidos no setor de neurologia da clínica escola da UNINASSAU-CG. Após a aceitação, foi agendado um dia para a avaliação dos participantes. Nesse momento, os indivíduos foram levados a uma sala reservada 
e a eles foram esclarecidas todas as informações relacionadas a pesquisa. Após os esclarecimentos 0 participante foi orientado a ler e, em caso de aceitação, realizar a assinatura do Termo de Consentimento Livre e Esclarecido (TCLE).

Em seguida os participantes foram submetidos ao protocolo de avaliação, que foi composto incialmente por um questionário sócio demográfico, seguido da aplicação do Mini exame do estado mental (MEEM). Logo após foi aplicado o teste Timed Up \& Go (TUG) que avalia a mobilidade funcional, seguido do teste de equilíbrio de Berg. A avaliação ocorreu em dois momentos distintos, pré e pós aplicação do protocolo de pesquisa. O primeiro momento se sucedeu no início da pesquisa (um dia antes da primeira sessão) e o segundo momento logo após a realização das 10 sessões de atendimento (dia seguinte a décima sessão).

Para a coleta de dados os pacientes foram divididos aleatoriamente em dois grupos, Grupo 1 fisioterapia motora e prática mental (GFM + PM) e grupo 2 fisioterapia motora (GFM), ambos foram submetidos a 10 sessões de fisioterapia duas vezes na semana. O GFM realizava 45 minutos de fisioterapia motora e o GFM + PM foi submetido a fisioterapia motora associada a prática mental, sendo fisioterapia motora 30 minutos e prática mental 15 minutos.

O protocolo de FM, comum aos dois grupos, foi desenvolvido pelas pesquisadoras e foi composto por exercícios divididos em 3 posturas (deitado, sentado e em pé). Cada sessão de FM foi constituída por exercícios que 
incluíram: mobilizações articulares de tornozelo e pé com duração de 4 minutos cada, dissociação de cintura pélvica (3 minutos), exercícios resistidos com fortalecimento de complexo póstero lateral, adutores e quadríceps ambos com 3 séries de 10 repetições (com ênfase no membro inferior acometido), descarga de peso, mudança de decúbito de sentado para em pé, Treino equilíbrio estático (Romberg sensibilizado 2x30s, Semi tandem 2 vezes de 30 segundos (olhos abertos) e treino de marcha para frente e lateral ( 5 repetições cada).

No grupo GFM + PM as sessões de PM ocorreram em uma sala tranquila após a realização da fisioterapia motora. Durante a prática mental o paciente foi orientado a assumir posição ortostática, em seguida solicitado a identificar e sequenciar as articulações necessárias para a realização de um único passo, sendo eles: flexão da coxa e perna, extensão da perna, dorsiflexão do pé, toque do calcanhar, descarga de peso no pé e corpo inclinado à frente. Em todas as sessões o paciente foi instruído a buscar utilizar os membros inferiores de forma igualitária na execução da tarefa e realizar a imaginação do tipo visual.

A PM foi composta de três fases, na primeira o paciente descrevia verbalmente os componentes cinemáticos para realização do passo e executava-os. Na segunda etapa, falava os componentes e imaginava-os e na última etapa apenas imaginava os componentes cinemáticos. As fases um e dois do protocolo, objetivaram preparar o paciente para 
PM. Cada etapa foi executada em três séries de 10 repetições.

\section{Análise estatística}

Após a finalização da coleta de dados, estes foram armazenados e tratados no software Statistical Package for the Social Sciences (SPSS) versão 24 para Windows e apresentados por meio de tabelas. A estatística descritiva foi realizada por meio da exposição dos valores absolutos, relativos, médias e desvio-padrão. A comparação das variáveis se deu por meio do teste T-Student, e a normalidade da amostra foi verificada de acordo com o teste Kolmogorov-Smirnov. As análises levaram em consideração o nível de confiança de $95 \%$.

\section{RESULTADOS}

A presente pesquisa foi composta por 6 pacientes, do sexo masculino divididos em dois grupos GFM $(n=3)$ e GFM $+\operatorname{PM}(n=3)$. Na amostra estudada a idade média dos participantes foi de 56 13,91 anos, enquanto a média geral de tempo decorrido o AVC foi de 56 $\pm 28,05$ meses. De modo específico os pacientes do grupo GFM + PM tinham a média de idade de $53 \pm 11,53$ anos e uma média de tempo decorrido

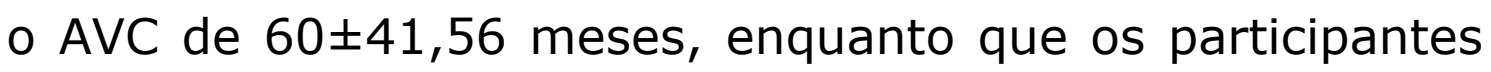
do grupo GFM tinham uma média de idade de $59 \pm 18,0$ anos e de tempo transcorrido o AVC de 52士13,85 meses. 
Em relação as variáveis "escolaridade", "presença de comorbidades" e "grau de independência para a realização das Atividades de Vida Diária - AVD's" pode-se observar a Tabela 1.

Tabela 1. Perfil sociodemográfico e clínico de indivíduos com AVC da clínica escola da UNINASSAU, Campina Grande, 2019.

\begin{tabular}{|c|c|c|c|c|}
\hline $\begin{array}{c}\text { Características sócio } \\
\text { demográficas }\end{array}$ & Grupo & & n (06) & $\%$ \\
\hline \multirow{8}{*}{ Escolaridade } & \multirow{4}{*}{ GFM + PM } & Analfabeto & 0 & 0 \\
\hline & & Fundamental & 2 & 33,3 \\
\hline & & Médio & 0 & 0 \\
\hline & & Superior & 1 & 16,7 \\
\hline & \multirow{4}{*}{ GFM } & Analfabeto & 1 & 16,7 \\
\hline & & Fundamental & 0 & 0 \\
\hline & & Médio & 1 & 16,7 \\
\hline & & Superior & 1 & 16,7 \\
\hline \multirow{6}{*}{ Comorbidades } & \multirow{3}{*}{ GFM + PM } & Desequilíbrio & 2 & 33,3 \\
\hline & & Diabetes & 1 & 16,7 \\
\hline & & Nenhuma & 0 & 0 \\
\hline & \multirow{3}{*}{ GFM } & Desequilíbrio & 1 & 16,7 \\
\hline & & Diabetes & 0 & 0 \\
\hline & & Nenhuma & 2 & 33,3 \\
\hline \multirow{4}{*}{ AVD's } & \multirow{3}{*}{ GFM + PM } & Independente & 1 & 16,7 \\
\hline & & $\begin{array}{l}\text { Semi } \\
\text { independente }\end{array}$ & 2 & 33,3 \\
\hline & & Independente & 2 & 33,3 \\
\hline & GFM & $\begin{array}{l}\text { Semi } \\
\text { independente }\end{array}$ & 1 & 16,7 \\
\hline
\end{tabular}

AVD's: Atividades da vida diária. GFM: Grupo Fisioterapia motora. GFM+PM: Grupo fisioterapia motora + prática mental.

Em relação a independência para a realização das AVD's pode-se observar que $50 \%$ dos participantes eram semi independentes. 
Como demonstra a Tabela 2, em relação aos valores do MEEM, os grupos apresentaram o escore maior que 21 não manifestando nenhum distúrbio cognitivo, tendo em vista que este foi um dos critérios de inclusão neste estudo. Observa-se também um aumento no escore para ambos os grupos entre a avaliação e a reavaliação.

Tabela 2. Valores obtidos nos instrumentos de avaliação dos indivíduos com AVCI da Clínica escola da UNINASSAU, Campina Grande, 2019.

\begin{tabular}{ccc}
\hline Instrumentos & $\begin{array}{c}\text { GFM + PM } \\
\text { (média } \pm \text { DP) }\end{array}$ & $\begin{array}{c}\text { GFM } \\
\text { (média } \pm \text { DP) }\end{array}$ \\
\hline MEEM (avaliação) & $25,67 \pm 1,52$ & $25,00 \pm 4,00$ \\
MEEM (reavaliação) & $27,67 \pm 1,52$ & $26,33 \pm 4,72$ \\
EEB (avaliação) & $49,67 \pm 2,30$ & $44,00 \pm 16,46$ \\
EEB (reavaliação) & $53,00 \pm 4,35$ & $45,00 \pm 14,73$ \\
TUG (avaliação) & $31,67 \pm 13,86$ & $39,00 \pm 24,75$ \\
TUG (reavaliação) & $26,67 \pm 14,57$ & $40,33 \pm 23,62$
\end{tabular}

DP: Desvio-Padrão. MEEM: Mini Exame do Estado Mental. EEB: Escala de Equilíbrio de Berg. TUG: Timed up and Go. GFM: Grupo Fisioterapia motora. GFM+PM: Grupo fisioterapia motora + prática mental.

Em relação a avaliação do equilíbrio, realizado por meio da EEB, observou-se que ambos os grupos apresentaram uma maior pontuação na reavaliação quando comparado a avaliação. Além disso, pode-se perceber que o grupo GFM + PM apresentou uma elevação no escore mais pronunciada. $A$ escala apresenta pontuação máxima de 56 pontos, em que um escore final de maior pontuação indica um bom equilíbrio e consequentemente um menor risco de quedas. Como 
demonstrado na Tabela 3, não houve diferença entre os grupos GFM+PM e GFM.

Tabela 3. Resultados obtidos na comparação entre grupos, Campina Grande, 2019.

\begin{tabular}{cccc}
\hline Variáveis & GFM + PM - GFM & T Student & Valor-p \\
& Média \pm DP & & \\
\hline MEEM (avaliação) & $0,667 \pm 2,47$ & 0,270 & 0,801 \\
MEEM (reavaliação) & $1,33 \pm 2,86$ & 0,465 & 0,666 \\
EEB (avaliação) & $7,00 \pm 9,78$ & 0,716 & 0,542 \\
EEB (reavaliação) & $8,00 \pm 8,86$ & 0,902 & 0,418 \\
TUG (avaliação) & $-7,33 \pm 16,38$ & $-0,448$ & 0,678 \\
TUG (reavaliação) & $-13,66 \pm 16,02$ & $-0,853$ & 0,442 \\
\hline
\end{tabular}

PM: Prática Mental. FM: Fisioterapia Motora. DP: Desvio-Padrão. MEEM: Mini Exame do Estado Mental. EEB: Escala de Equilíbrio de Berg. TUG: Timed up and Go.

\section{DISCUSSÃO}

Foi observada predominância de indivíduos do gênero masculino, ratificando estudos similares que demonstram que os homens são mais acometidos pelo AVC, principalmente indivíduos com mais de 50 anos, por serem mais expostos aos fatores de risco ${ }^{10,11}$. Ratificando um estudo que afirma que o AVC é uma doença que afeta predominantemente idosos ${ }^{12}$.

De forma contrária, um estudo mais recente relata que a população feminina vem sendo mais acometida. Ressaltase que essa população é mais vulnerável a doenças cardiovasculares e cerebrovasculares pois, além da ocupação profissional fora de casa, as mulheres são as principais responsáveis pelas tarefas domésticas. Essa fonte de 
estresse se associa ao fator idade e predispõe a um maior acometimento de AVC após a menopausa ${ }^{13}$.

Em estudos anteriores, a idade média dos participantes foi de 60 anos, de ambos os sexos, porém com predominância do sexo masculino, e o tempo transcorrido o AVC apresentava-se entre 1 a 48 meses $^{9,14}$. O tempo de lesão é bastante variável entre os estudos, há relato de tempo de lesão de 36 meses $^{15}$, e estudo que utilizou pacientes com 8 meses após o evento lesivo ${ }^{16}$. Outros estudos não descreveram o perfil dos pacientes e entre eles houve uma grande variação entre o tempo após o $A V C^{8,17}$.

Vale ressaltar a importância da reabilitação durante a fase aguda do AVC pois influencia positivamente 0 prognóstico funcional dos pacientes. Neste sentido 0 propósito é iniciar a reabilitação o mais precocemente possível, para a prevenção de complicações relacionadas a imobilização prolongada no leito, complicações pulmonares e a restauração da funcionalidade ${ }^{18}$. A reabilitação precoce influencia na recuperação da capacidade funcional de realizar tarefas, auxilia na reabilitação motora, devolve a autonomia do paciente e contribui na autoestima3,19.

Em relação a independência para a realização das atividades de vida diária (AVD's) pode-se observar que 50\% dos participantes eram semi independentes reafirmando um estudo prévio que relata que o AVC é a segunda principal causa de incapacidade, deixando inúmeras sequelas físicas, restringindo a funcionalidade do indivíduo principalmente nas $A V D ' s^{20}$. 
De acordo com a mensuração da mobilidade e o risco de quedas, avaliado por meio do TUG, foi possível observar que o grupo GFM + PM teve uma diminuição da pontuação relacionada a avaliação, enquanto o grupo GFM teve um aumento. Esse resultado mostra que o grupo GFM + PM apresentou uma evolução na realização do teste, visto que quanto menor for o valor final melhores são as respostas, sugerindo um melhor equilíbrio e menor risco de quedas. Em buscas realizadas em bases de dados não foram encontrados estudos que relatem sobre a influência da prática mental em relação as variáveis mensuradas pelo TUG.

Em um estudo prévio foi utilizada a PM associada a prática física e foi comparada as fases pré e pós-tratamento. Os achados alcançados afirmam que a PM associada à prática física pode reduzir a incapacidade e melhorar a função dos membros de pacientes pós-AVC crônico ${ }^{15}$.

Foram encontrados artigos nos quais em sua maioria utilizaram a PM como ferramenta para a recuperação da função do membro superior afetado15,21. De modo geral os estudos encontrados demonstram vantagens a aplicação da prática mental no AVC em relação ao membro superior acometido, relatando melhora na amplitude de movimento de punho e melhora na força de preensão ${ }^{22}$.

E em relação aos membros inferiores e a marcha, são descritas melhoras na descarga de peso dinâmica no lado hemiparético e melhora no posicionamento do centro de gravidade ${ }^{23}$. A PM também pode ser usada para a melhora do equilíbrio estático e dinâmico. Foi relatado em um estudo 
conduzido com 17 indivíduos pós AVC um aumento significativo na velocidade média da marcha e no tamanho dos passos após seis semanas de intervenção com a PM24. Os integrantes receberam treinamentos com supervisão domiciliar durante 15 a $20 \mathrm{~min}$.

Em estudo conduzido na Coréia do Sul investigou-se o efeito da PM sobre a marcha, por meio de uma análise comparativa entre grupo experimental e grupo controle ${ }^{25}$. No grupo experimental, os pacientes realizaram um treinamento com prática mental de treino de marcha usando um vídeo, além da fisioterapia convencional. Já no grupo controle, os pacientes realizaram a visualização de um documentário, além de fisioterapia convencional. O treinamento foi realizado 5 vezes por semana, durante 4 semanas. O estudo identificou que o grupo experimental apresentou um aumento da velocidade da marcha quando comparado ao grupo controle.

Em um estudo piloto utilizando a prática mental na doença de Parkinson, em que os participantes foram divididos em dois grupos (experimental - GE e controle GC) ${ }^{26}$. O GE realizou 15 sessões de fisioterapia motora 2 vezes na semana por 40 minutos, seguidos de 15 minutos de PM. Os participantes do GC receberam o mesmo atendimento, exceto a PM. Os resultados mostraram que o grupo que realizou a PM associada a fisioterapia convencional obteve uma melhora mais evidente em relação ao risco de quedas, avaliado pelo teste TUG e Teste de caminhada de 10 minutos. Para a monitorização da realização da PM e de 
modo a potencializar o engajamento do paciente, os pesquisadores utilizaram um eletrodo conectado a um eletroencefalógrafo, porém apesar de estarem visualizando o aparelho ligado, a monitorização não estava sendo executada (efeito sham).

É importante reforçar que ainda são escassos os estudos que abordam a utilização da prática mental como recurso associado a fisioterapia motora. Os estudos previamente levantados analisaram um número pequeno de indivíduos, dificultando respostas significativas para o uso da PM no AVC, além disso as intervenções eram distintas e utilizavam escalas de avaliação diferentes, o que torna difícil a comparação do presente estudo com os demais.

Não houve diferença entre os grupos GFM+PM e GFM, entretanto, observou-se que os dois grupos apresentaram melhoras o que sugere que o indivíduo pós AVC pode ser beneficiado na recuperação do equilíbrio e na redução do risco de quedas realizando apenas a fisioterapia motora ou realizando a pratica mental associada a fisioterapia motora.

Diante disto, a falta de garantia de que o paciente realmente execute a PM é uma das limitações mais importantes para a utilização dessa técnica. No entanto, mesmo que os indivíduos tenham uma boa capacidade de imaginação há sempre a incerteza e incapacidade de controlar a execução do indivíduo, tanto em frequência quanto à forma como ele imagina os movimentos relacionados à tarefa motora? . 
Também foi levantada a incerteza quanto aos efeitos que podem ocorrer em pacientes muito comprometidos e a necessidade de que o paciente já tenha alguma habilidade prévia em realizar a tarefa a ser treinada com a PM, ou seja, alguma representação interna do movimento, para que os resultados sejam satisfatórios ${ }^{27}$.

Relata-se que a quantidade de prática parece ser realmente importante para a melhora da capacidade de imaginação a ser obtida. Porém, devido à escassez de estudos que tenham investigado a capacidade da imaginação, não se sabe quanto tempo de treinamento seria necessário para melhorar essa capacidade dos indivíduos ${ }^{28}$.

Diante disso pode-se observar que a prática mental parece ser uma técnica complementar que não substitui a execução motora dos movimentos, capaz de proporcionar efeitos adicionais ao treinamento motor. Como ponto positivo observa-se a segurança de sua execução e a dispensa de instalações especiais e equipamentos, sendo um recurso simples e de baixo custo ${ }^{29}$.

A prática mental associada à fisioterapia parece promover bons resultados na reabilitação motora, mas, ainda existe a carência na adesão da técnica por parte dos profissionais, pelo desconhecimento, por falta de um número maior de comprovação cientifica e por ser uma terapia que necessita de tempo de planejamento por parte do fisioterapeuta e muitas repetições por parte do paciente para que se obtenham bons resultados. 


\section{CONCLUSÃO}

Ainda que o presente estudo apresente limitações, como o número reduzido de participantes, foi possível identificar que o grupo que realizou a prática mental associada a fisioterapia apresentou uma melhor evolução do equilíbrio do que o grupo que realizou apenas a fisioterapia. Entretanto, reforça-se que esses resultados não foram significativos.

Além disso, reforçamos a importância da adesão do fisioterapeuta a novas técnicas, visando não só um tratamento convencional voltado para fisioterapia musculoesquelética, mas sim interligar os sistemas envolvidos na ativação de áreas corticais relacionadas a realização do movimento que são de extrema importância para a estimulação da plasticidade neuronal.

\section{AGRADECIMENTOS}

Agradecimento aos voluntários da pesquisa e ao Centro Universitário Maurício de Nassau (UNINASSAU - Campina Grande - PB).

\section{REFERÊNCIAS}

1.Barros LA, Passos NRS, Runes MASN. Breve estudo do estado da arte sobre acidente vascular cerebral e serious games para aplicação no projeto "AVC' do núcleo de tecnologia assistida da UFS. Rev Geitec 2013:3:129-43. https://doi.org/10.7198/geintec.v3i1.94

2.Arrais JSL, Lima AM, Silva TG. Atuação dos profissionais fisioterapeutas na reabilitação do paciente vítima de acidente vascular encefálico. Rev Interdiscip 2016;9:179-84.

https://dialnet.unirioja.es/servlet/articulo?codigo $=6772018$ 
3.O'Sullivan SB, Schmiti TJ, Susan B. Fisioterapia: Avaliação e tratamento. São Paulo: Barueri, 2010.

https://www.saraiva.com.br/fisioterapia-avaliacao-e-tratamento-5ed-2010-2869418/p

4.Smith EE, Soposnik G, Biessels GJ, Doubal FN, Forage M, Kasner SE, et al. Prevention of Stroke in Patients with Silent Cerebrovascular Disease: A Scientific Statement for Healthcare Professionals from the American Heart Association/American Stroke Association. J Am Heart Assoc 2016;48: e44-71.

https://doi.org/10.1161/STR.0000000000000116

5.Woellner SS, Araujo AGS, Cabral FMH, Uessler PNP, Soares AV. Testes de Equilíbrio em pacientes hemiparéticos por AVC. Neurociencias (RJ) 2015;11:32-40.

http://docplayer.com.br/68247571-Testes-de-equilibrio-em-

pacientes-hemipareticos-por-avc.html

6.Gaspar BE, Hotta TTH, Souza LAPSS. Prática mental na reabilitação de membro superior após acidente vascular encefálico: casos clínicos. Conscientização Saúde 2011;10:319-32.

https://doi.org/10.5585/conssaude.v10i2.2483

7.Sant'anna LF, Guida S, Silva JG. Informações Somatossensoriais nos Processos da Prática Mental na Fisioterapia Neurofuncional: Estudo de Revisão. Rev Neurocienc 2014;22:95-101.

https://doi.org/10.34024/rnc.2014.v22.8123

8. Jackson PL, Lafleur MF, Malouin F, Richards C, Doyon J. Potencial role of mental practice using motor imagery in neurologia rehabilitation. Arc Phys Med Reab 2001;82:1133-41.

https://doi.org/10.1053/apmr.2001.24286

9.Sharma N, Pomeroy VM, Baron JC. Motor imagery: a backdoor to the motor system after stroke? Stroke 2006;37:1941-52.

https://doi.org/10.1161/01.STR.0000226902.43357.fc

10.Nadruz WJR. Diagnóstico e tratamento dos fatores de risco. Rev Com Ciência 2009;109:1-3.

http://comciencia.scielo.br/pdf/cci/n109/a17n109.pdf

11.Araújo JS, Silva SED, Santana ME, Vasconcelos EV, Conceição VMA. Obrigação de (des) cuidar: representações sociais sobre o cuidado a sequelados de acidente vascular cerebral por seus cuidadores. Rev Min Enferm 2011;1698-105. http://www.reme.org.br/artigo/detalhes/506 12.Luna ACP. Avaliação da capacidade funcional em pacientes internados em uma unidade de AVC isquêmico no interior do ceará utilizando as escalas de Barthel e Rankin. In: Congresso brasileiro de doenças cerebrovasculares. Anais de epidemiologia. Fortaleza-CE, Brasil. 2013. http://www.avc2013.com.br/

13. Araújo MC, Silva MBF, Ponte KMA. Conhecimentos e risco para acidente vascular cerebral em mulheres. SANARE 2018;17:6-12. https://doi.org/10.36925/sanare.v17i2.1256

14.Yoo $E$, Chung B. The effect of visual feedback plus mental practice on symmetrical weight-bearing training in people with hemiparesis. Clin Rehabil 2006;20:388-97.

https://doi.org/10.11.91/026.9215506cr962oa 
15.Page SJ, Szaflarski JP, Eliassen JC, Pan H, Cramer SC. Cortical plasticity following motor skill learning during mental practice in stroke. Neurorehabil Neural Repair 2009;32:382-8. https://doi.org/10.1177/154596.8308326427

16.Butler AJ, Page SJ. Mental practice with motor imagery: evidence for motor recovery and cortical reorganization after stroke. Arch Phys Med Rehabil 2006;87(Suppl 2):S2-11.

https://doi.org/10.1016/j.apmr.2006.08.326

17.Braun SM, Beurskens AJ, Borm PJ, Schack T, Wade DT. The effects of mental practice in stroke rehabilitation: a systematic review. Arch Phys Med Rehabil 2012;87:842-52.

https://doi.org/10.1016/j.apms.2006.02.034

18.Oujamaaa L, Marquera A, Franconyb G, Davoinea P, Chrispina A, Payenb JF, et al. Intérêt d'une rééducation précoce pour les patients neurologiques. Ann Franç Anesth Réanim 2012;31:e253-63. https://doi.org/10.1016/j.amfar.2012.08.005

19.Silva DCS, Nascimento CF, Brito ES. Efeitos da Mobilização Precoce nas Complicações Clínicas Pós-AVC: Revisão da Literatura. Rev Neurocienc 2013;21:620-7.

https://doi.org/10.4181/RNC.2013.21.891.8p

20.Rangel ESS, Belasco AGS, Diccini S. Quality of life of patients with stroke rehabilitation. Acta Paul Enferm 2013;26:205-12. https://doi.org/10.1590/S0103-21002013000200016

21. Hewett $T E$, Ford $K R$, Levine $P$, Page SJ. Reaching kinematics to measure motor changes after mental practice in stroke. Top Stroke Rehabil 2009;14:23-9. http://dx.doi.org/10.1310/tsr1404-23

22.Dijkerman HC, Ietswaart $M$, Johnston $M$, Macwalter RS. Does motor imagery training improve hand function in chronic stroke patients? A pilot study. Clin Rehabil 2015;18:538-49.

http://dx.doi.org/10.1191/0269215504cr769oa

23.Yoo E, Park E, Chung B. Mental practice effect on line-tracing accuracy in persons with hemiparetic stroke: a preliminary study. Arch Phys Med Rehabil 2001;82:1213-8.

http://dx.doi.org/10.1053/apmr.2001.25095

24.Dunsk A, Dickstein R, Marcovitz E, Levy S, Deutsch JE. Home-based motor imagery training for gait rehabilitation of people with chronic post stroke hemiparesis. Arch Phys Med Rehabil 2008;89:1580-8. http://dx.doi.org/10.1016/j.apmr.2007.12.039

25. Hwang $\mathrm{S}$, Jeon $\mathrm{H}$, Syi $\mathrm{CH}$, Kwon OY, Cho $\mathrm{SH}$, You SH. Locomotor imagery training improves gait performance in people with chronic hemiparetic stroke: a controlled clinical trial. Clin Rehabil 2010; 24:514-22. https://doi.org/10.1177/026915509360645

26.Silva LP, Duarte MP, Souza CB, Lins CS, Coriolano MG, Lins OG. Efeitos da prática mental associada a fisioterapia motora sobre a marcha e o risco de quedas na doença de Parkinson: estudo piloto. Fisioter Pesqui 2019;26:112-9. https://dx.doi.org/10.1590/1809$\underline{2950 / 17012926022019}$ 
27.Vries SD, Mulder T. Motor imagery and stroke rehabilitation: a critical discussion. J Rehabil Med 2011;39:5-13. http://dx.doi.org/10.2340/16501977-0020 28.Stefanello JMF, Marques CP, Rodacki ALF. Assessment of motor imagery ability and training. Rev Bras Cineantropom Desemp Hum 2010;12:395-400. http://dx.doi.org/10.1590/S198000372010000600001.

29.Andrade TG, Asa SKP. Prática Mental para Pacientes com Sequelas Motoras Pós Acidente Vascular Cerebral. Rev Neurocienc 2011;19:54250. https://doi.org/10.34024/rnc.2011.v19.8354 